\title{
Effects of an intensive lifestyle intervention and the role of sleep in people living with HIV and prediabetes: a pilot and feasibility study
}

\author{
Hataikarn Nimitphong ${ }^{1,7^{*}}$ (D), Somnuek Sungkanuparph ${ }^{2}$, Chatvara Areevut ${ }^{1}$, Sunee Saetung ${ }^{1}$, \\ Ratanaporn Jerawatana ${ }^{3}$, Amornrat Hathaidechadusadee ${ }^{4}$, Supaporn Somwang ${ }^{4}$, Wanabhorn Tongchom ${ }^{5}$, \\ Nampeth Saibuathong ${ }^{3}$, Jandanee Sakmanarit ${ }^{1}$, Orawan Pichitchaipitak ${ }^{3}$, Angsana Phuphuakrat ${ }^{1}$ and \\ Sirimon Reutrakul ${ }^{6}$
}

\begin{abstract}
Objectives: Prediabetes is prevalent in people living with HIV (PLWH). Insufficient and irregular sleep are linked to abnormal glucose metabolism. This study aimed to investigate the differences in sleep characteristics between PLWH with and without prediabetes, determine the acceptability/feasibility and effects of a pilot six-month intensive lifestyle intervention (ILI) program on glucose metabolism in those with prediabetes, and determine how sleep modulates these effects.

Results: Thirty-nine PLWH (20 normoglycemia and 19 prediabetes) participated. There were no differences in sleep characteristics between individuals with normoglycemia and prediabetes. Next, thirteen individuals with prediabetes completed a six-month ILI program. The ILI program resulted in significant body weight reduction at 6 months $(63.5 \pm 13.9$ to $61.9 \pm 14.0 \mathrm{~kg}, p=0.012)$, which was maintained at 12 months $(p<0.001)$. Waist circumferences were significantly decreased at 12 months $(85.4 \pm 11.7$ to $82.9 \pm 12.7 \mathrm{~cm}, p=0.014)$. An increase in sleep variability was significantly associated with an increase in 2-h plasma glucose, independent of changes in BMI ( $b=0.603)$, and physical activity $(b=0.774)$. This pilot study suggested that ILI in PLWH with prediabetes is feasible and effective in improving metabolic control, with its effects possibly modulated by sleep variability. These findings should be confirmed in a larger study to reduce diabetes risk in this population.
\end{abstract}

Trail registration: ClinicalTrial.gov, NCT03545217 (date of registration: May 22, 2018)

Keywords: HIV, Intensive lifestyle intervention, Prediabetes, Sleep

\section{Introduction}

Type 2 diabetes mellitus (T2DM) is prevalent in people living with HIV (PLWH) $[1,2]$. In Thailand, the prevalence of T2DM and prediabetes among PLWH are $14 \%$ [3] and $27.5 \%$ [4], respectively. The prediabetes

\footnotetext{
*Correspondence: hataikarnn@hotmail.com; hataikarn.nim@mahidol.ac.th ${ }^{1}$ Department of Medicine, Faculty of Medicine Ramathibodi Hospital, Mahidol University, Bangkok, Thailand

Full list of author information is available at the end of the article
}

prevalence in this group was higher than the $10.6 \%$ rate in the general Thai population [5].

Intensive lifestyle interventions (ILI) are effective in reducing diabetes rates and are generally recommended to the general population and PLWH with prediabetes [6-8]. Whether or not the unique characteristics of PLWH affects the outcomes is unknown. To date, there have been only a few studies that explored the effectiveness of ILI in PLWH with impaired glucose tolerance. A non-randomized study of $28 \mathrm{PLWH}$ that had impaired 
fasting glucose showed that ILI for 6 months improved glucose, blood pressure and lipids levels [9]. Another study showed that a supplementation of pioglitazone to an exercise program for 4 months improved insulin sensitivity in PLWH that had insulin resistance and central obesity [10]. Therefore, understanding the acceptability and efficacy of ILI along with factors that may modulate the responses, is crucial in reducing the diabetes risk in this group.

In people with normoglycemia, prediabetes and T2DM, sleep disturbances, including insufficient sleep, poor sleep quality and increased sleep variability (varying day-to-day sleep timing or duration), have been linked to abnormal glucose metabolism [11] and cardiometabolic risks [12-14]. Studies in non-HIV people have shown that prediabetes individuals had shorter sleep durations $(<5-6$ hours $(\mathrm{h}) /$ night $)[15,16]$ when compared to normoglycemic individuals. When compared to nonHIV infected people, PLWH had a higher prevalence of poor sleep quality $[17,18]$. However, there has been no study exploring the differences in sleep characteristics between PLWH with normoglycemia vs. prediabetes. ILI could potentially improve sleep. For example, exercise has been shown to improve sleep quality in patients with insomnia [19] and fibromyalgia [20]. The potential mechanisms include thermoregulation, body restoration, energy conservation hypotheses [21], and regulation of pro-inflammatory cytokines [22] in response to exercise. In addition, weight loss could improve obstructive sleep apnea severity which could improve sleep quality and duration [23]. There has only been limited studies on whether or not sleep characteristics modulate the response of diabetes prevention interventions.

The objectives of this study were to investigate the differences in sleep characteristics between PLWH with and without prediabetes. Furthermore, we tested the feasibility and effects of a pilot six-month ILI program on glucose metabolism in those with prediabetes and explored whether sleep characteristics modulated the response to ILI. The acceptability of the program was evaluated. The results of this study will provide information to design future larger studies aimed at reducing diabetes risks in PLWH with prediabetes.

\section{Main text}

\section{Materials and methods}

This study was conducted among PLWH who received antiretroviral therapy (ART) and were being followed at an infectious disease clinic in a university hospital in Bangkok, Thailand. Thirty-nine PLWH (20 normoglycemia and 19 prediabetes) from a previous cross-sectional study for the prevalence of prediabetes among PLWH [24], who were willing to participate, were included in this study. Prediabetes was defined as follows: hemoglobin A1c $(\mathrm{HbA} 1 \mathrm{c}) \geq 5.7-<6.5 \%$, fasting plasma glucose (FPG) levels $<126 \mathrm{mg} / \mathrm{dL}$, and 2-h plasma glucose (2 h-PG) levels $<200 \mathrm{mg} / \mathrm{dL}$ following a 75 -g oral glucose tolerance test (OGTT) [25]. Exclusion criteria included medical problems causing limited physical activities (for example; active coronary artery disease, obstructive lung disease) [24, 26], the use of glucose lowering medications, known obstructive sleep apnea, performing shift work and pregnancy. This study adhered to CONSORT guidelines.

\section{Study protocol}

This study consisted of two parts. In the first part, a cross-sectional study, 39 PLWH (20 normoglycemia and 19 prediabetes) were assessed for their medical histories and anthropometric measurements. OGTT, HbA1c, and objective sleep assessments were performed (see below).

In the second part, a pilot interventional study of a single open trial, 14 of 19 individuals with prediabetes (74\%) participated in a six-month ILI program. Repeated anthropometric measurements and glucose homeostasis were performed at the end of the program and at 12 months in $13 \mathrm{PLWH}$ with prediabetes who completed the ILI program. Sleep patterns were reassessed at 6 months.

\section{Glucose homeostasis assessment}

After an overnight fast, 75-g of glucose was given orally. Blood samples were obtained at $0 \mathrm{~min}$ for $\mathrm{HbAlc}$, and at $0,30,60,90$, and $120 \mathrm{~min}$ for glucose and insulin. To evaluate early changes in glucose homeostasis, the markers of glucose metabolism, including the Matsuda index [27], HOMA-IR [28], the insulinogenic index [29] and the Disposition Indices [30] were calculated.

\section{Objective and subjective sleep assessment}

Individuals wore an Actiwatch 2 wrist activity monitor (Philips Respironics, Bend, Oregon) for 7 days. Sleep duration was defined as the amount of actual sleep obtained at night, and sleep efficiency as a percentage of time in bed spent sleeping. Mid-sleep time (MST) was defined as the midpoint between sleep start and wake time. The standard deviations (SDs) of sleep duration and MST were calculated and used as sleep variability parameters as previously described [13]. For each individual, the mean across all available nights was used.

Sleep quality in the previous month was assessed using a validated Thai version of the Pittsburgh Sleep Quality Index (PSQI) [31]. 


\section{Physical activity assessment}

The physical activity was assessed by the Global Physical Activity Questionnaire (GPAQ) version 2 and measured as Metabolic Equivalents (METs) [32,33].

\section{Quality of life}

Quality of life was measured using the European Quality of Life Measure-5 Domain-5-Level (EQ-5D-5L) [34, 35] and the visual analog scale [VAS; scale ranged from 0 (the worst) to 100 (the best)].

\section{The six-month intensive lifestyle intervention (ILI) program}

This pilot six-month ILI program was led by a multidisciplinary team of diabetes nurses and dieticians, and included a follow up at 12 months. The program was a group-based activity adapted from the Diabetes Prevention Program (DPP)-Thailand project, which was shown to be effective in improving glycemic parameters [36]. The goals of the program were to target three main aspects of a healthy lifestyle including healthy eating (aiming 7\% weight loss in overweight individuals), exercise (150 min of aerobic exercise weekly) and healthy coping (for example, practicing in spiritual and mindfulness, emotional management). This modified program consisted of five one-day sessions which met every six weeks over the 6-month period, and supplementary weekly telephone follow-ups (10-15 minutes). Each session included instructions from the health care team, through workshop activities and the setting of mutually-agreed behavioral goals. In-between-visit telephone follow-ups were aimed at encouraging goal achievement, discussion of problems and enhancing health knowledge.

To evaluate the acceptability of the intervention, questionnaires which addressed the participants' satisfaction of their experience were administered 4 times, at the end of each session (1, 2, 3 and 4).

\section{Statistical analysis}

Comparisons of the differences between normoglycemia and prediabetes groups were performed by Student t Test, Mann-Whitney U test and Pearson's chi-squared test, as appropriate. Comparison of metabolic parameters at baseline and 6 months, and baseline and 12 months, were performed by paired-samples T-test or Wilcoxon Signed Ranks Test. Spearman's correlation was used to investigate the association between changes in body mass index (BMI), physical activity, sleep parameters and changes in metabolic parameters at 6 months compared to baseline. A multiple linear regression analysis was further performed to investigate the association between changes in sleep parameters and changes in metabolic parameters after adjusting for the changes in BMI and physical activity. Analyses were performed using SPSS statistical software package, version 18.0 (SPSS, Chicago IL, USA).

\section{Results}

Baseline demographics, metabolic and sleep characteristics in normoglycemic and prediabetic individuals

Thirty-nine PLWH (20 normoglycemia and 19 prediabetes) with a mean age of $51.5 \pm 6.0$ years were included (Table 1). There were no differences in clinical characteristics between the two groups (Table 1). As expected, individuals with prediabetes had higher FPG, 2 h-PG, HbA1c, and HOMA-IR than those in the normoglycemia group. Other indices obtained from OGTT (Matsuda, Insulinogenic and Disposition Indices), lipid profiles and all sleep parameters were similar between the two groups.

\section{Results of ILI}

Thirteen PLWH with prediabetes completed the sixmonth ILI program and a 12-month follow-up visit. At 6 months, there was a significant reduction in BW and BMI from baseline, which was maintained at 12 months (Table 2). Waist circumferences (WC) significantly decreased at 12 months (Table 2). There were no changes in physical activity during the follow-up period. FPG non-significantly decreased at 6 months $(p=0.051)$, whereas there were no changes in $2 \mathrm{~h}-\mathrm{PG}$ and HbA1c at 6 and 12 months. For OGTT-derived indices, when compared to baseline, the Matsuda index non-significantly increased at 12 months $(p=0.064)$, HOMA-IR significantly decreased at both 6 and 12 months, and an insulinogenic index non-significantly decreased at 6 months $(p=0.055)$.

For sleep parameters, sleep efficiency (a marker of sleep quality) significantly increased at 6 months, whereas there were no changes in other parameters (Table 2).

Quality of life assessed by EQ-5D-5L did not change. However, quality of life as assessed by VAS significantly increased at 6 months.

\section{Relationship between changes in BMI, physical activity, sleep parameters and changes in metabolic parameters during ILI}

We further investigated the association between changes in BMI, physical activity, sleep parameters and changes in metabolic parameters at 6 months (Table 3). A decrease in BMI correlated to an increase in the Matsuda index. An increase in physical activity was associated with a reduction in $\mathrm{HbA1c}$ and insulinogenic index. For changes in sleep parameters, an increase in sleep duration was associated with a reduction in $\mathrm{HbA} 1 \mathrm{c}$ and insulinogenic 
Table 1 Baseline clinical characteristics of 20 PLWH with normoglycemia and 19 PLWH with prediabetes

\begin{tabular}{|c|c|c|c|}
\hline Characteristics & $\begin{array}{l}\text { Normoglycemia } \\
(n=20)\end{array}$ & $\begin{array}{l}\text { Prediabetes } \\
(n=19)\end{array}$ & $P$ value \\
\hline \multicolumn{4}{|c|}{ Demographic and anthropometric parameters } \\
\hline Age (years) & $51.8 \pm 6.6$ & $51.2 \pm 5.5$ & 0.767 \\
\hline Male gender, number (\%) & $13(65)$ & $12(63.2)$ & 1.00 \\
\hline Family history of diabetes, number (\%) & $4(20)$ & $9(47.4)$ & 0.096 \\
\hline History of smoking, number (\%) & $10(50)$ & $6(31.6)$ & 0.333 \\
\hline History of alcohol drinking, number (\%) & 16(80) & $11(57.9)$ & 0.176 \\
\hline \multicolumn{4}{|l|}{ Underlying diseases, number (\%) } \\
\hline Dyslipidemia & $9(45)$ & 7(36.8 & 0.748 \\
\hline Hypertension & $4(20)$ & $3(15.8)$ & 1.00 \\
\hline NAFLD & 0 & $1(5.3)$ & 0.487 \\
\hline Cancer & 0 & 0 & NA \\
\hline Others $^{\mathrm{a}}$ & 0 & 0 & NA \\
\hline BW (kg) & $62.7 \pm 12.8$ & $63.2 \pm 12.5$ & 0.912 \\
\hline $\mathrm{BMI}\left(\mathrm{kg} / \mathrm{m}^{2}\right)$ & $23.1 \pm 4.6$ & $24.1 \pm 4.1$ & 0.500 \\
\hline $\mathrm{SBP}(\mathrm{mmHg})$ & $130.5 \pm 15.5$ & $130.1 \pm 14.7$ & 0.935 \\
\hline $\mathrm{DBP}(\mathrm{mmHg})$ & $81.3 \pm 8.1$ & $82.1 \pm 10.9$ & 0.781 \\
\hline WC (cm) & $84.8 \pm 10.8$ & $85.3 \pm 11$ & 0.901 \\
\hline Neck circumference (cm) & $35 \pm 3.1$ & $35.0 \pm 4.0$ & 0.947 \\
\hline \multicolumn{4}{|l|}{ HIV-related parameters } \\
\hline Duration of HIV infection (years) & $15.2 \pm 6.1$ & $14.5 \pm 5.5$ & 0.729 \\
\hline $\begin{array}{l}\text { Type of ART regimen, number (\%) } \\
\text { NNRTI-based } \\
\text { PI-based } \\
\text { Others }\end{array}$ & $\begin{array}{l}13(65) \\
7(35) \\
0\end{array}$ & $\begin{array}{l}13(68.4) \\
6(31.6) \\
0\end{array}$ & $\begin{array}{l}1.00 \\
1.00 \\
\text { NA }\end{array}$ \\
\hline Duration of ART (years) & $11.5 \pm 6.1$ & $11.9 \pm 5.3$ & 0.843 \\
\hline CD4 cell counts (cells/mm³) & $559.8 \pm 264$ & $455.7 \pm 190.7$ & 0.169 \\
\hline \multicolumn{4}{|l|}{ Metabolic and other biochemical parameters } \\
\hline $\mathrm{FPG}(\mathrm{mg} / \mathrm{dL})$ & $91.8 \pm 7.9$ & $99.1 \pm 14.9$ & 0.002 \\
\hline $2 \mathrm{~h}-\mathrm{PG}(\mathrm{mg} / \mathrm{dL})$ & $113.5 \pm 40$ & $153.6 \pm 46.9$ & 0.007 \\
\hline $\mathrm{HbA1c}(\%)$ & $5.32 \pm 0.21$ & $5.99 \pm 0.23$ & $<0.001$ \\
\hline Matsuda Index & $3.29(1.73-5.41)$ & $2.44(1.53-3.44)$ & $0.160^{b}$ \\
\hline HOMA-IR & $2.51(1.19-3.04)$ & $3.66(1.74-6.02)$ & $0.044^{b}$ \\
\hline Insulinogenic Index & $0.79(0.11-1.52)$ & $0.84(0.34-1.51)$ & $0.901^{b}$ \\
\hline Disposition Index & $2.65(0.29-5.32)$ & $1.70(0.84-3.66)$ & $0.550^{\mathrm{b}}$ \\
\hline Alanine transaminase $(U / L)$ & $36.6 \pm 18.5$ & $38.8 \pm 21.5$ & 0.729 \\
\hline Total cholesterol (mg/dL) & $219 \pm 42$ & $204.3 \pm 37$ & 0.255 \\
\hline HDL cholesterol (mg/dL) & $51.2 \pm 11.6$ & $46.8 \pm 11.3$ & 0.248 \\
\hline LDL cholesterol (mg/dL) & $141.3 \pm 36$ & $130.6 \pm 34.2$ & 0.350 \\
\hline Triglycerides (mg/dL) & $146.1 \pm 90.8$ & $166 \pm 122.2$ & 0.566 \\
\hline \multicolumn{4}{|l|}{ Sleep parameters } \\
\hline PSQI & $5(3-7)$ & $4(3-7)$ & $0.607^{b}$ \\
\hline Sleep efficiency (\%) & $81.9(78.2-85.9)$ & $84.0(77.6-87.8)$ & $0.627^{b}$ \\
\hline Sleep duration (min) & $338.5(316.3-367.5)$ & $354.6(332.8-388.9)$ & $0.283^{b}$ \\
\hline SD sleep duration (min) & $52.9(33.9-72.6)$ & $51.3(34.9-76.0)$ & $0.708^{b}$ \\
\hline SD MST & $0: 42 \pm 0: 30$ & $0: 30 \pm 0: 18$ & 0.123 \\
\hline
\end{tabular}

Data was presented as mean \pm SD or IQR

$P L W H$ people living with HIV, BW body weight, BMI body mass index, NAFLD nonalcoholic fatty liver disease, $A R T$ antiretroviral therapy, NNRTI non-nucleoside reverse transcriptase inhibitor, $P$ I protease inhibitor, SBP systolic blood pressure, DBP diastolic blood pressure, WC waist circumference, $F P G$ fasting plasma glucose, $2 \mathrm{~h}-P G$ 2-h plasma glucose, HbA1c hemoglobin A1c, HDL high-density lipoprotein, LDL low-density lipoprotein, PSQI Pittsburgh Sleep Quality Index, SD standard deviation, MST mid-sleep time

a Including cerebrovascular disease, coronary artery disease and chronic kidney disease

b Mann Whitney U test

c time presented as 24-h clock time 
index. However, the associations became non-significant after controlling for changes in BMI or physical activity. In addition, an increase in sleep variability (SD sleep duration and SD MST) was significantly associated with an increase in $2 \mathrm{~h}-\mathrm{PG}$. The association between $\triangle \mathrm{SD}$ sleep duration and $2 \mathrm{~h}$-PG remained significant after adjusting for changes in BMI $(b=0.603,95 \% \mathrm{CI}$ : 0.146 , $1.059, p=0.015)$ or physical activity $(\mathrm{b}=0.774,95 \% \mathrm{CI}$ : $0.339,1.209, p=0.003)$, but the $\Delta$ SD MST and 2 h-PG became non-significant after adjustment.

This ILI program was well-accepted. Over $90 \%$ of participants were satisfied. The program attendance was $84.6-100 \%$, suggesting that the program was feasible in this setting.

\section{Discussion}

In this pilot study of 20 normoglycemic and 19 prediabetic PLWH, we did not find differences in sleep characteristics between the two groups. The pilot six-month ILI program for PLWH with prediabetes was effective in improving diabetes risks, including a reduction in BW, BMI, WC and HOMA-IR, which were maintained at 12 months. The program was feasible and acceptable, as evaluated by the participants' attendance and satisfaction. Furthermore, the changes in sleep variability were a novel factor associated with changes in metabolic parameters after ILI. An increase in SD sleep duration was significantly associated with increased 2 h-PG regardless of the changes in BMI and physical activity. These results from the present study suggest that ILI is effective and feasible in reducing diabetes risks in PLWH with prediabetes, and that sleep modification could possibly be complimentary to ILI. Therefore, a larger dedicated RCT should be performed to confirm these findings.

Of note, the magnitude of BW reductions in the present study was smaller than the pre-specified goal of the program. However, this was comparable to the findings from a Community-Based DPP in Thailand [36]. This might be due to the lower baseline weight. Nevertheless, the weight reduction was accompanied by a significant reduction in HOMA-IR over the 12-month period.

This is the first study demonstrating that objectively measured sleep duration and sleep variability may play a role in the effectiveness of ILI in PLWH. However, the findings should be interpreted with caution due to the small number of subjects. Insufficient sleep demonstrated to increase diabetes risk in some studies [11,37]. Furthermore, maintaining adequate sleep was also shown to be beneficial during a weight loss study [38]. In a study of ILI, individuals with prediabetes who reported $\leq 6 \mathrm{~h} /$ night of sleep at enrollment had a significantly higher rate of incident diabetes, as well as less weight loss [39], compared to those sleeping $7 \mathrm{~h}$. Our results in PLWH are in agreement with this data, as we demonstrated that a decrease in objectively measured sleep duration was associated with an increase in HbA1c. In addition, this is the first study that demonstrated that increased sleep variability was associated with an increase in 2 h-PG after ILI. Increasing ten minutes of SD sleep duration increased $2 \mathrm{~h}$-PG $6 \mathrm{mg} / \mathrm{dL}$ after adjusting for changes in BMI, and increased $2 \mathrm{~h}-\mathrm{PG} 7.7 \mathrm{mg} / \mathrm{dL}$ after adjusting for changes in physical activity. Sleep variability has been increasingly recognized to be related to metabolic health. This could potentially be related to the shift in timing of sleep which could affect the body's circadian regulation, and hence, affect metabolism. Sleep variability has been shown to be associated with glycemic control in people with type 1 diabetes [13, 14], less physical activity [40], higher prevalence and incidence of metabolic abnormalities [41], and an unhealthy diet [42]. Our pilot results suggested that maintaining adequate and regular sleep could potentially be beneficial in reducing diabetes risks during ILI in PLWH.

In conclusion, intensive lifestyle interventions in PLWH with prediabetes is feasible and effective in improving metabolic control. The preliminary results suggest that the effects of ILI are modulated by sleep duration and sleep variability. This highlights a possibility to apply the ILI program and sleep adjustment as a diabetic prevention program in this high-risk group for T2DM.

\section{Limitation}

We recruited only PLWH in this study, therefore, we lacked a comparison of the differences in sleep variability and glucose metabolism in individuals without HIV. In this study, participants in normoglycemia and prediabetes groups were not matched, however, the demographic and anthropometric parameters were similar. In addition, we recruited PLWH with prediabetes who received ART with complete viral suppression. Thus, our results may not be applicable to PLWH without successful ART. Since the cultural and socioeconomic needs vary between different ethnic groups, the results may not be implied for non-Asian populations. The number of study participants was small. Lastly, more detailed objective sleep and metabolic measurements such as body fat composition, hyperinsulinemic euglycemic clamp and OSA assessment were not performed in this study." 
Table 2 Compared parameters at baseline, 6 months (at the end of the program) and 12 months in 13 PLWH with prediabetes

\begin{tabular}{|c|c|c|c|c|c|}
\hline Characteristics & baseline & 6 months & $\begin{array}{l}P \text { value } \\
\text { (baseline and } \\
6 \text { months) }\end{array}$ & 12 months & $\begin{array}{l}P \text { value } \\
\text { (baseline } \\
\text { and } \\
12 \text { months) }\end{array}$ \\
\hline
\end{tabular}

\section{Demographic parameters}

Age (years)

Male gender, number (\%)

$52.9 \pm 4.8$

Family history of diabetes, number (\%)

$8(61.5)$

History of smoking, number (\%)

$7(53.8)$

$4(30.8)$

$8(61.5)$

Underlying diseases, number (\%)

$\begin{array}{ll}\text { Dyslipidemia } & 4(30.5) \\ \text { Hypertension } & 2(15.4)\end{array}$

\section{HIV-related parameters}

Duration of HIV infection (years)

Type of ART regimen, number (\%)

NNRTI-based
PI-based
Others
Duration of ART (years)

CD4 cell counts (cells $/ \mathrm{mm}^{3}$ )

Anthropometric parameters and physical activity

BW $(\mathrm{kg})$
$\mathrm{BMI}\left(\mathrm{kg} / \mathrm{m}^{2}\right)$
$\mathrm{SBP}(\mathrm{mmHg})$
$\mathrm{DBP}(\mathrm{mmHg})$
WC $(\mathrm{cm})$
Neck circumference $(\mathrm{cm})$
Physical activity (MET)

\section{Metabolic parameters}

$$
\text { FPG (mg/dL) }
$$

$2 \mathrm{~h}-\mathrm{PG}(\mathrm{mg} / \mathrm{dL})$

HbA1c (\%)

Matsuda Index

HOMA-IR

Insulinogenic Index

Disposition Index

Total cholesterol (mg/dL)

$\mathrm{HDL}$ cholesterol (mg/dL)

LDL cholesterol (mg/dL)

Triglycerides (mg/dL)

\section{Sleep parameters}

PSQI

Sleep efficiency (\%)

Sleep duration (min)

SD sleep duration (min)

SD MST

Quality of life

EQ-5D-5L

VAS
5(38.5)

2(15.4)

1(7.7)

$15.3 \pm 4.9$

$8(61.5)$

$5(38.5)$

0

$12.7 \pm 5.5$

$405.1 \pm 157.0$

$8(61.5)$

$5(38.5)$

0
$466.6 \pm 239.3$

$63.5 \pm 13.9$

$24.3 \pm 4.3$

$131.6 \pm 16.2$

$82.9 \pm 12.6$

$85.4 \pm 11.7$

$35.1 \pm 4.7$

920 (60-2040)

$102 \pm 16.4$

$151.5 \pm 49.7$

$6.00 \pm 0.25$

2.53(1.45-5.33)

$3.72(1.35-10.11)$

0.84(0.39-1.90)

$1.70(0.88-4.26)$

$208.4 \pm 41.2$

$48.2 \pm 11.4$

$130.5 \pm 40.4$

$179.2 \pm 145.6$

$4.31 \pm 2.98$

$83.34 \pm 7.50$

$351.86 \pm 44.37$

$54.30 \pm 23.62$

$0: 26 \pm 0: 13$

$0.977 \pm 0.033$

$81.08 \pm 11.98$

$0: 34 \pm 0: 26$

$0.994 \pm 0.014$

$91.15 \pm 9.61$

$86.50 \pm 5.73$

$\begin{array}{ll}0.317 & 5(38.5) \\ 1 & 2(15.4) \\ 1 & 2(15.5)\end{array}$

$61.9 \pm 14.0 \quad 0.012$

0.014

0.025

0.531

0.110

0.568

0.173

1520 (420-3744)

0.051

0.406

0.676

$0.311^{b}$

$0.010^{\mathrm{b}}$

$0.055^{b}$

$0.152^{b}$

0.420

0.105

0.682

0.684

0.151

0.048

0.244

0.546

0.167

0.143

$8(61.5)$

5(38.5)

0

$459.8 \pm 238.2$

0.213

$62.0 \pm 14.3$

$<0.001$

$23.7 \pm 4.4$

$132.0 \pm 16.9$

$<0.001$

$82.3 \pm 10.1$

0.901

$82.9 \pm 12.7$

0.859

$34.2 \pm 4.6$

0.014

840 (130-1060)

0.025

0.442

$100.8 \pm 21.7 \quad 0.684$

$141.1 \pm 52 \quad 0.377$

$5.89 \pm 0.37$

0.103

7.49(1.42-11.85)

$0.064^{b}$

$1.51(0.44-4.10)$

$0.036^{\mathrm{b}}$

$0.52(0.33-1.74)$

$0.972^{b}$

$2.22(0.83-5.52)$

$0.311^{b}$

$208.8 \pm 52.9$

0.965

$51.2 \pm 17.1$

0.258

$134.0 \pm 45.7 \quad 0.679$

$135.8 \pm 60.9 \quad 0.274$

NA

NA

NA

NA

NA

NA

NA

NA

NA

NA

0.012

$0.945 \pm 0.064$

0.116

$83.46 \pm 8.26$

0.417 
Table 2 (continued)

Data was presented as mean $\pm \mathrm{SD}$ or IQR; ${ }^{\mathrm{a}}, \mathrm{n}=12{ }^{\mathrm{b}}{ }^{\mathrm{b}}$, Wilcoxon Signed Ranks Test; ${ }^{\mathrm{c}}$, time presented as 24 -h clock time

$P L W H$ people living with HIV, $B W$ body weight, $B M I$ body mass index, NAFLD nonalcoholic fatty liver disease, ART antiretroviral therapy, NNRTI non-nucleoside reverse transcriptase inhibitor, $P I$ protease inhibitor, SBP systolic blood pressure, DBP diastolic blood pressure, WC waist circumference, MET Metabolic Equivalents, $F P G$ fasting plasma glucose, 2 h-PG 2-h plasma glucose, HbA1c hemoglobin A1c, HDL high-density lipoprotein, LDL low-density lipoprotein, PSQ/ Pittsburgh Sleep Quality Index, SD standard deviation, MST mid-sleep time, EQ-5D-5L the European Quality of Life Measure-5 Domain-5-Level, VAS visual analog scale

${ }^{a} \mathrm{n}=12$

${ }^{\mathrm{b}}$ Wilcoxon Signed Ranks Test

c Time presented as 24-h clock time

Table 3 Correlation between changes in BMI, physical activity, sleep parameters and changes in metabolic parameters between baseline and 6 months in 13 PLWH with prediabetes

\begin{tabular}{|c|c|c|c|c|c|c|c|}
\hline & $\triangle \mathrm{FPG}$ & $\Delta 2 \mathrm{~h}-\mathrm{PG}$ & $\Delta \mathrm{HbA} 1 c^{\mathrm{a}}$ & $\Delta$ Matsuda index & $\triangle \mathrm{HOMA} I \mathrm{R}$ & $\Delta$ insulinogenic index & $\Delta$ disposition index \\
\hline$\triangle \mathrm{BMI}$ & 0.505 & 0.495 & 0.322 & $-0.560^{*}$ & 0.258 & 0.264 & -0.214 \\
\hline$\triangle \mathrm{MET}$ & 0.076 & 0.190 & $-0.634^{*}$ & -0.019 & 0.212 & $-0.595^{*}$ & -0.424 \\
\hline$\Delta$ Sleep efficiency & -0.287 & -0.283 & -0.231 & 0.005 & -0.346 & -0.176 & -0.088 \\
\hline$\Delta$ Sleep duration & 0.097 & 0.113 & $-0.587^{*}$ & -0.044 & -0.192 & $-0.571^{*}$ & -0.214 \\
\hline$\triangle S D$ Sleep duration & -0.168 & $0.790^{* *}$ & 0.000 & 0.363 & 0.374 & 0.137 & -0.016 \\
\hline$\triangle S D M S T$ & 0.127 & $0.597^{*}$ & -0.098 & 0.148 & -0.016 & -0.269 & -0.132 \\
\hline
\end{tabular}

Bold indicated statistically significant results

PLWH people living with HIV, FPG fasting plasma glucose, 2 h-PG 2-h plasma glucose, HbA1c hemoglobin A1c, BMI body mass index, SD standard deviation, MST midsleep time

a $\mathrm{n}=12 ;{ }^{*}, \mathrm{p}<0.05 ;{ }^{* *}, \mathrm{p}<0.01$

\section{Abbreviations}

2 h-PG: 2-H plasma glucose; ART: Antiretroviral therapy; BMl: Body mass index; DPP: Diabetes Prevention Program; EQ-5D-5L: The European Quality of Life Measure-5 Domain-5-Level; FPG: Fasting plasma glucose; GPAQ: Global Physical Activity Questionnaire; h: Hour; HbA1c: Hemoglobin A1c; HIV: Human Immunodeficiency Virus; ILI: Intensive lifestyle interventions; METs: Metabolic Equivalents; MST: Mid-sleep time; OGTT: Oral glucose tolerance test; PSQI: Pittsburgh Sleep Quality Index; RCT: Randomized controlled trial; SDs: Standard deviations; T2DM: Type 2 diabetes mellitus; VAS: Visual analog scale; WC: Waist circumference.

\section{Acknowledgements}

We gratefully acknowledge the assistance of Mr. Brian Daniels for English editing and proofreading. We sincerely thank Asst.Prof. Valla Tantayotai RN, D.N.S and Dr. Sakawduan Numsangkul, MD for their guidance.

\section{Authors' contributions}

HN, SS (Somnuek Sungkanuparph) and SR conceived of the study, participated in its design and coordination, performed the statistical analysis and were major contributors in writing the manuscript. CA, SS (Sunee Saetung), RJ, AH, SS (Supaporn Somwang), WT, NS, JS, and OP conducted the ILI program. AP conceived of the study design and participated in its design. All authors read and approved the final manuscript.

\section{Funding}

This work was supported by Grants from the Faculty of Medicine Ramathibodi Hospital, Mahidol University and the Thailand Research Fund (grant number RTA6080009).

\section{Availability of data and materials}

The datasets used and/or analyzed during this study are available from the corresponding author on reasonable request.

\section{Declarations}

Ethics approval and consent to participate

Ethics approvals were obtained from the institutional review boards, Faculty of Medicine Ramathibodi Hospital, Mahidol University, Bangkok, Thailand. The informed written consent was obtained from participants prior to enrollment.

\section{Consent for publication}

Not applicable

\section{Competing interests}

HN received speaker honoraria from Novo Nordisk, Takeda, MSD, Sanofi Aventis, Amgen, Eli lily, Boehringer Ingelheim Pharmaceuticals and Novartis. SR received speaker honoraria from Novo Nordisk, Sanofi Aventis and Medtronic; a research grant from Merck Sharp and Dohme; and non-financial support from ResMed, Thailand. SS, CA, SS, RJ, AH, SS, WT, NS, JS, OP and AP report no conflicts of interest.

\section{Author details}

${ }^{1}$ Department of Medicine, Faculty of Medicine Ramathibodi Hospital, Mahidol University, Bangkok, Thailand. ${ }^{2}$ Chakri Naruebodindra Medical Institute, Faculty of Medicine Ramathibodi Hospital, Samut Prakan, Thailand. ${ }^{3}$ Nursing Department, Faculty of Medicine Ramathibodi Hospital, Mahidol University, Bangkok, Thailand. ${ }^{4}$ Division of Nutrition and Dietetics, Faculty of Medicine Ramathibodi Hospital, Mahidol University, Bangkok, Thailand. ${ }^{5}$ Nutrition Science Group, Faculty of Medicine Ramathibodi Hospital, Mahidol University, Bangkok, Thailand. ${ }^{6}$ Division of Endocrinology, Diabetes and Metabolism, University of Illinois At Chicago, Chicago, IL, USA. ${ }^{7}$ Division of Endocrinology and Metabolism, Department of Medicine, Faculty of Medicine Ramathibodi Hospital, Mahidol University, Rama VI Road, Ratchathewi, Bangkok 10400, Thailand.

Received: 11 September 2020 Accepted: 8 April 2021

Published online: 17 April 2021 


\section{References}

1. Tripathi A, Jerrell JM, Liese AD, Zhang J, Rizvi AA, Albrecht H, et al. Association of clinical and therapeutic factors with incident dyslipidemia in a cohort of human immunodeficiency virus-infected and non-infected adults: 1994-2011. Metab Syndr Relat Disord. 2013;11(6):417-26.

2. Tripathi A, Liese AD, Jerrell JM, Zhang J, Rizvi AA, Albrecht $H$, et al. Incidence of diabetes mellitus in a population-based cohort of HIV-infected and nonHIV-infected persons: the impact of clinical and therapeutic factors over time. Diabet Med. 2014:31(10):1185-93.

3. Chantrathamachart P, Sungkanuparph S, Kiertburanakul S, Malathum K. Diabetes mellitus and hypertension in hiv-infected patients receiving antiretrovial therapy: a pilot study. J Infect Dis Antimicrob Agent. 2006;22:131.

4. Srivanich N, Ngarmukos C, Sungkanuparph S. Prevalence of and risk factors for pre-diabetes in HIV-1-infected patients in Bangkok, Thailand. J Int Assoc Physicians AIDS Care (Chic). 2010;9(6):358-61.

5. Aekplakorn W, Chariyalertsak S, Kessomboon P, Sangthong R, Inthawong R, Putwatana $\mathrm{P}$, et al. Prevalence and management of diabetes and metabolic risk factors in Thai adults: the Thai National Health Examination Survey IV, 2009. Diabetes Care. 2011;34(9):1980-5.

6. Knowler WC, Barrett-Connor E, Fowler SE, Hamman RF, Lachin JM, Walker EA, et al. Reduction in the incidence of type 2 diabetes with lifestyle intervention or metformin. N Engl J Med. 2002;346(6):393-403.

7. American Diabetes A. 3. Prevention or Delay of Type 2 Diabetes: Standards of Medical Care in Diabetes-2019. Diabetes Care. 2019:42(Suppl 1):S29-S33.

8. Brown TT, Glesby MJ. Management of the metabolic effects of HIV and HIV drugs. Nat Rev Endocrinol. 2011;8(1):11-21.

9. Duncan AD, Peters BS, Rivas C, Goff LM. Reducing risk of Type 2 diabetes in HIV: a mixed-methods investigation of the STOP-Diabetes diet and physical activity intervention. Diabet Med. 2020;37(10):1705-14

10. Yarasheski KE, Cade WT, Overton ET, Mondy KE, Hubert S, Laciny E, et al. Exercise training augments the peripheral insulin-sensitizing effects of pioglitazone in HIV-infected adults with insulin resistance and central adiposity. Am J Physiol Endocrinol Metab. 2011;300(1):E243-51.

11. Anothaisintawee T, Reutrakul S, Van Cauter E, Thakkinstian A. Sleep disturbances compared to traditional risk factors for diabetes development: systematic review and meta-analysis. Sleep Med Rev. 2016;30:11-24.

12. Zhou M, Lalani C, Banda JA, Robinson TN. Sleep duration, timing, variability and measures of adiposity among 8- to 12-year-old children with obesity. Obes Sci Pract. 2018;4(6):535-44.

13. Chontong S, Saetung S, Reutrakul S. Higher sleep variability is associated with poorer glycaemic control in patients with type 1 diabetes. J Sleep Res. 2016:25(4):438-44

14. Patel NJ, Savin KL, Kahanda SN, Malow BA, Williams LA, Lochbihler G, et al. Sleep habits in adolescents with type 1 diabetes: variability in sleep duration linked with glycemic control. Pediatr Diabetes. 2018;19(6):1100-6.

15. Engeda J, Mezuk B, Ratliff S, Ning Y. Association between duration and quality of sleep and the risk of pre-diabetes: evidence from NHANES. Diabet Med. 2013;30(6):676-80

16. Lou P, Chen P, Zhang L, Zhang P, Chang G, Zhang N, et al. Interaction of sleep quality and sleep duration on impaired fasting glucose: a populationbased cross-sectional survey in China. BMJ Open. 2014:4(3):e004436.

17. Gutierrez J, Tedaldi EM, Armon C, Patel V, Hart R, Buchacz K. Sleep disturbances in HIV-infected patients associated with depression and high risk of obstructive sleep apnea. SAGE Open Med. 2019;7:2050312119842268.

18. Huang X, Li H, Meyers K, Xia W, Meng Z, Li C, et al. Burden of sleep disturbances and associated risk factors: a cross-sectional survey among HIV-infected persons on antiretroviral therapy across China. Sci Rep. 2017:7(1):3657.

19. Banno M, Harada Y, Taniguchi M, Tobita R, Tsujimoto H, Tsujimoto $Y$, et al. Exercise can improve sleep quality: a systematic review and meta-analysis. PeerJ. 2018;6:e5172.

20. Estevez-Lopez F, Maestre-Cascales C, Russell D, Alvarez-Gallardo IC, Rodriguez-Ayllon M, Hughes CM, et al. Effectiveness of exercise on fatigue and sleep quality in fibromyalgia: a systematic review and meta-analysis of randomized trials. Arch Phys Med Rehabil. 2020.

21. Berger RJ, Phillips NH. Comparative physiology of sleep, thermoregulation and metabolism from the perspective of energy conservation. Prog Clin Biol Res. 1990:345:41-50 (discussion 1-2)
22. Driver HS, Taylor SR. Exercise and sleep. Sleep Med Rev. 2000;4(4):387-402.

23. Roche J, Isacco L, Masurier J, Pereira B, Mougin F, Chaput JP, et al. Are obstructive sleep apnea and sleep improved in response to multidisciplinary weight loss interventions in youth with obesity? A systematic review and meta-analysis. Int J Obes (Lond). 2020;44(4):753-70.

24. Phuphuakrat A, Nimitphong H, Reutrakul S, Sungkanuparph S. Prediabetes among HIV-infected individuals receiving antiretroviral therapy: prevalence, diagnostic tests, and associated factors. AIDS Res Ther. 2020;17(1):25.

25. American Diabetes A. 2. Classification and diagnosis of diabetes: standards of medical care in diabetes-2019. Diabetes Care. 2019;42(Suppl 1):S13-S28.

26. Pedersen BK, Saltin B. Evidence for prescribing exercise as therapy in chronic disease. Scand J Med Sci Sports. 2006;16(Suppl 1):3-63.

27. Matsuda M, DeFronzo RA. Insulin sensitivity indices obtained from oral glucose tolerance testing: comparison with the euglycemic insulin clamp. Diabetes Care. 1999;22(9):1462-70.

28. Matthews DR, Hosker JP, Rudenski AS, Naylor BA, Treacher DF, Turner RC. Homeostasis model assessment: insulin resistance and beta-cell function from fasting plasma glucose and insulin concentrations in man. Diabetologia. 1985;28(7):412-9.

29. Seltzer HS, Allen EW, Herron AL Jr, Brennan MT. Insulin secretion in response to glycemic stimulus: relation of delayed initial release to carbohydrate intolerance in mild diabetes mellitus. J Clin Invest. 1967:46(3):323-35.

30. Weiss R, Cali AM, Dziura J, Burgert TS, Tamborlane WV, Caprio S. Degree of obesity and glucose allostasis are major effectors of glucose tolerance dynamics in obese youth. Diabetes Care. 2007;30(7):1845-50.

31. Sitasuwan T, Bussaratid S, Ruttanaumpawan P, Chotinaiwattarakul W. Reliability and validity of the Thai version of the Pittsburgh Sleep Quality Index. J Med Assoc Thai. 2014;97(Suppl 3):S57-67.

32. Bull FC, Maslin TS, Armstrong T. Global physical activity questionnaire (GPAQ): nine country reliability and validity study. J Phys Act Health. 2009:6(6):790-804

33. Anothaisintawee T, Lertrattananon D, Thamakaison S, Thakkinstian A, Reutrakul S. The relationship among morningness-eveningness, sleep duration, social jetlag, and body mass index in asian patients with prediabetes. Front Endocrinol (Lausanne). 2018;9:435.

34. Tongsiri S, Cairns J. Estimating population-based values for EQ-5D health states in Thailand. Value Health. 2011;14(8):1142-5.

35. Pattanaphesaj J. Health-related quality of life measure (EQ-5D-5L): measurement property testing and its preference-based score in Thai population [Doctoral dissertation]: Mahidol University; 2014

36. Aekplakorn W, Tantayotai V, Numsangkul S, Tatsato N, Luckanajantachote P, Himathongkam T. Evaluation of a community-based diabetes prevention program in Thailand: a cluster randomized controlled trial. J Prim Care Community Health. 2019;10:2150132719847374.

37. Reutrakul S, Van Cauter E. Interactions between sleep, circadian function, and glucose metabolism: implications for risk and severity of diabetes. Ann NY Acad Sci. 2014:1311:151-73.

38. Chaput JP, Tremblay A. Sleeping habits predict the magnitude of fat loss in adults exposed to moderate caloric restriction. Obes Facts. 2012;5(4):561-6.

39. Nuyujukian DS, Beals J, Huang H, Johnson A, Bullock A, Manson SM, et al. Sleep duration and diabetes risk in american indian and alaska native participants of a lifestyle intervention project. Sleep. 2016;39(11):1919-26.

40. Duncan MJ, Kline CE, Rebar AL, Vandelanotte C, Short CE Greater bed- and wake-time variability is associated with less healthy lifestyle behaviors: a cross-sectional study. Z Gesundh Wiss. 2016;24(1):31-40.

41. Huang T, Redline S. Cross-sectional and prospective associations of actigraphy-assessed sleep regularity with metabolic abnormalities: The Multi-Ethnic Study of Atherosclerosis. Diabetes Care. 2019:42(8):1422-9.

42. Kjeldsen JS, Hjorth MF, Andersen R, Michaelsen KF, Tetens I, Astrup A, et al. Short sleep duration and large variability in sleep duration are independently associated with dietary risk factors for obesity in Danish school children. Int J Obes (Lond). 2014:38(1):32-9.

\section{Publisher's Note}

Springer Nature remains neutral with regard to jurisdictional claims in published maps and institutional affiliations. 